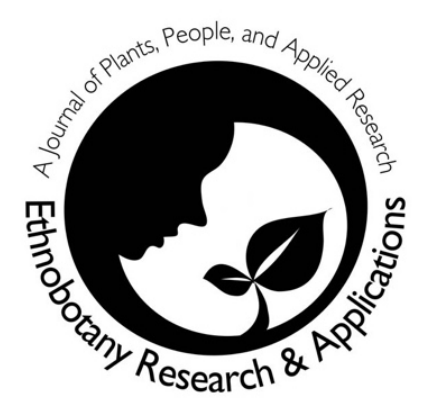

\title{
Ethnobotanical Survey of Important Wild Medicinal Plants of Tehsil Gojra, District Toba Tek Singh, Punjab, Pakistan
}

\author{
Muhammad Tufail, Khalid Hussain, Khalid Nawaz, Khizar \\ Hayat Bhatti, Ghulam Yasin and Syed Saqib Ali
}

\section{Databases and Inventories}

\begin{abstract}
Background: Almost every region of the world is stacked with a large number of medicinal plants. Medicinal plants are the major source to cure many diseases among the peoples of the world. There are still many areas in the world which have not been explored for its flora. Pakistan is also a rich with variety of flora but still there are some areas which need to be studied. This study was conducted to enlist the wild ethnomedicinally important plant species and traditional medicinal knowledge of Gojra, district Toba Tek Singh, Punjab, Pakistan.
\end{abstract}

Methods: Field surveys were arranged to enlist the important medicinal plants and traditional knowledge from the local community through questionnaire and group discussion during 2019-2020. Data collected through questionnaire was consist of plant local name, medicinal use, method of use and part used.

Results: There were more than 100 plant species including cultivated and wild but only 47 wild plants species were ethnomedicinally important as reported by local respondents that were placed in 20 families. It was noted that leaves had highest percentage $(54 \%)$ of its use in various prescriptions. Acacia modesta Wall. (Fabaceae) was the most common tree used in teeth pain and gastritis with 0.73 UV (Use Value) and 0.075 RFC (Relative Frequency of Citation) values. Achyranthes aspera L. (Amaranthaceae) showed 0.85 UV and 0.088 RFC values and was used to cure fever, asthma and cough. For stomach, gastric and urinary disorders, Chenopodium album L. (Amaranthaceae) was used with 0.78 UV and 0.156 RFC values. Cichorium intybus L. (Asteraceae) has much importance in summer season as it is commonly used to reduce thirst in hot weather conditions. Its UV was 0.74 and RFC was 0.132. Leaves, fruits and barks of Dalbergia sissoo DC. (Fabaceae) was used to control nosebleed, gonorrhoea, stop vomiting and skin disorder. It has 0.72 UV and 0.075 RFC values. Eucalyptus globulus Labill. (Myrtaceae) was used to treat common cold, respiratory infections and asthma. Its leaves were used to provide steam to the asthma patients. It showed $0.76 \mathrm{UV}$ and $0.08 \mathrm{RFC}$ values. Ricinus communis L. (Euphorbiaceae) was a common weed in the area with 0.74 UV and 0.19 RFC values. Its seeds and leaves were useful to treat rheumatic pain, joint pain and constipation. Acacia modesta showed the highest Fidelity Level (FL) value that was $87 \%$ and used in teeth and gastric problems.

Conclusions: It was concluded that there are many useful wild plants in Tehsil Gojra, district Toba Tek Singh, Punjab, Pakistan that are used in traditional medicines to cure many diseases. These plants also showed pharmacological importance that can be utilized to develop medicines.

Keywords: Gojra, ethnobotany, flora, survey

\section{Correspondence}

Muhammad Tufail ${ }^{1}$, Khalid Hussain ${ }^{1^{*}}$, Khalid Nawaz ${ }^{1}$, Khizar Hayat Bhatti ${ }^{1}$, Ghulam Yasin ${ }^{2}$ and Syed Saqib Ali ${ }^{1}$

${ }^{1}$ University of Gujrat, Gujrat, Pakistan

${ }^{2}$ Bahauddin Zakariya University, Multan, Pakistan

Corresponding Author: khalid.hussain@uog.edu.pk

Ethnobotany Research \& Applications

20:23 (2020)

\section{Background}

Ethnomedicinal studies are of great importance to discover contemporary drugs from indigenous medicinal plants (Njoroge et al., 2004). It is the appropriate source to collect the useful medicinal information and documentation (Mahmood et al., 2013). Ethnomedicinal documentation of traditional/ 
folk knowledge of native plants have much contributed for many vital drugs discoveries (Gilani and Rehman, 2005). It is estimated that about $25 \%$ of herbal drugs in modern pharmacopeia are plant based (Umair et al., 2017). Ethnomedicinal studies showed that almost all the plants reported in any area have medicinal importance. These plants are not only used in folk medicinal but also provide to health care units to manufacture many useful medicines (Njoroge et al., 2004).

Plants are providing reliable therapy since ancient time throughout the world. Almost every region of the world is stacked with a large number of medicinal plants. Pakistan has e great diversity in medicinal plants and people use these plants in various ethnomedicine to cure many diseases (Alamgeer et al., 2018). Pakistan is diverse in weather, terrestrial areas, conventional zones and flora (Hussain et al., 2008). Pakistan has predominantly huge, spotted and various diversity of medicinal plants. Roughly 6000 medicinal plants are verified from Kashmir and Pakistan (Shinwari, 1996). Khan et al. (2019) described that medicinal plants have been used from the very beginning in health care systems and these plants have shown the efficacy in various diseases.

Medicinal plants have not only importance among local community but currently have much more importance in the Western society (Shinwari and Gilani, 2003). In the world about 50,000 plants are used for medicinal purpose (Cunningham, 2014). Most of the rural community depends upon locally available plants to cure many diseases (Hussain et al., 2010). Many researchers have documented the medicinal importance of local flora in various regions of the world including Pakistan (Rehman et al., 2020). Similarly, Ali et al. (2020) studied the important weeds with their medicinal significance of district Gujrat, Pakistan.

The wild medicinal flora of Tehsil Gojra has not been studied. Few studies are available related to weeds of crops. So, there is a need to provide the useful medicinal information of the flora. This study was designed to document the wild flora and its ethnomedicinal values among the local inhabitant of Tehsil Gojra, district Toba Tek Singh, Punjab, Pakistan.

\section{Materials and methods}

\section{Study site}

Present study was conducted in Tehsil Gojra, district Toba Tek Singh, Punjab Pakistan during mid-season of summer and winter of 2019-2020. Tehsil Gojra, district Toba Tek Singh is situated at the latitude of $31^{\circ} 25^{\prime} \mathrm{N}$ and at longitude of $73^{\circ} 20^{\prime} \mathrm{E}$. City is located at $710 \mathrm{ft}$ asl (above sea level). Tehsil Gojra is joined on the eastern side with the district Faisalabad and in the west, it has district Jhang (Figure 1). North of Tehsil Gojra is linked with district Chiniot and in the south with district Toba Tek Singh (Muhammad et al., 2009).

There were total 40 visits and each visit was of 5 days to collect the plants and information from total 20 study sites nearby rural areas where local people depend upon wild plants to cure various diseases. Plants were collected with the help of local guiders who were familiar about the area. Those plants were selected which have mature leaves, stem, roots and preferable flowers. All the wild angiosperm plants were collected and only those plants were further investigated which were used by local people in medicine. The plant samples were collected and dried to mount on herbarium sheets that were identified with distinctive field guiders with their local names. A probabilistic approach was used considering simple and stratified random sampling methods (Levy and Lemeshow, 2008).

\section{Data collection}

Information about common name of the plants and part used for a specific disease were collected through interviews and questionnaires. There were 100 respondents ( $2 \%$ of the local residents) based upon by gender, age and occupation. Respondent were from different age ranging from 20-75 years including male, female and local health providers (hakims). There were 68 male and 32 females respondent with different age group i.e. 18 between 20-30 years of age, 22 between 31-40 years, 20 were 41-50 age group, 17 from 51-60, 17 from 61-70 and 6 respondents were $>70$ years of age. Among the respondents, $45 \%$ were traditional healers, $40 \%$ laypersons and $15 \%$ teachers and other professional. Questionnaire was designed to collect the desired ethnomedicinal information. There were four types main key informant i.e. hakims, plant providers, growers and students that were interviewed for local name, part used, medicinal use and method. Plants were identified with the help of reference plants preserved at herbarium, Department of Botany, University of Gujrat, Pakistan and the collected plants specimens have been deposited in this existing herbarium and further it was cross verified through flora of Pakistan (Nasir and Ali, 1983). Voucher specimens were deposited at Govt. Postgraduate College Gojra. 


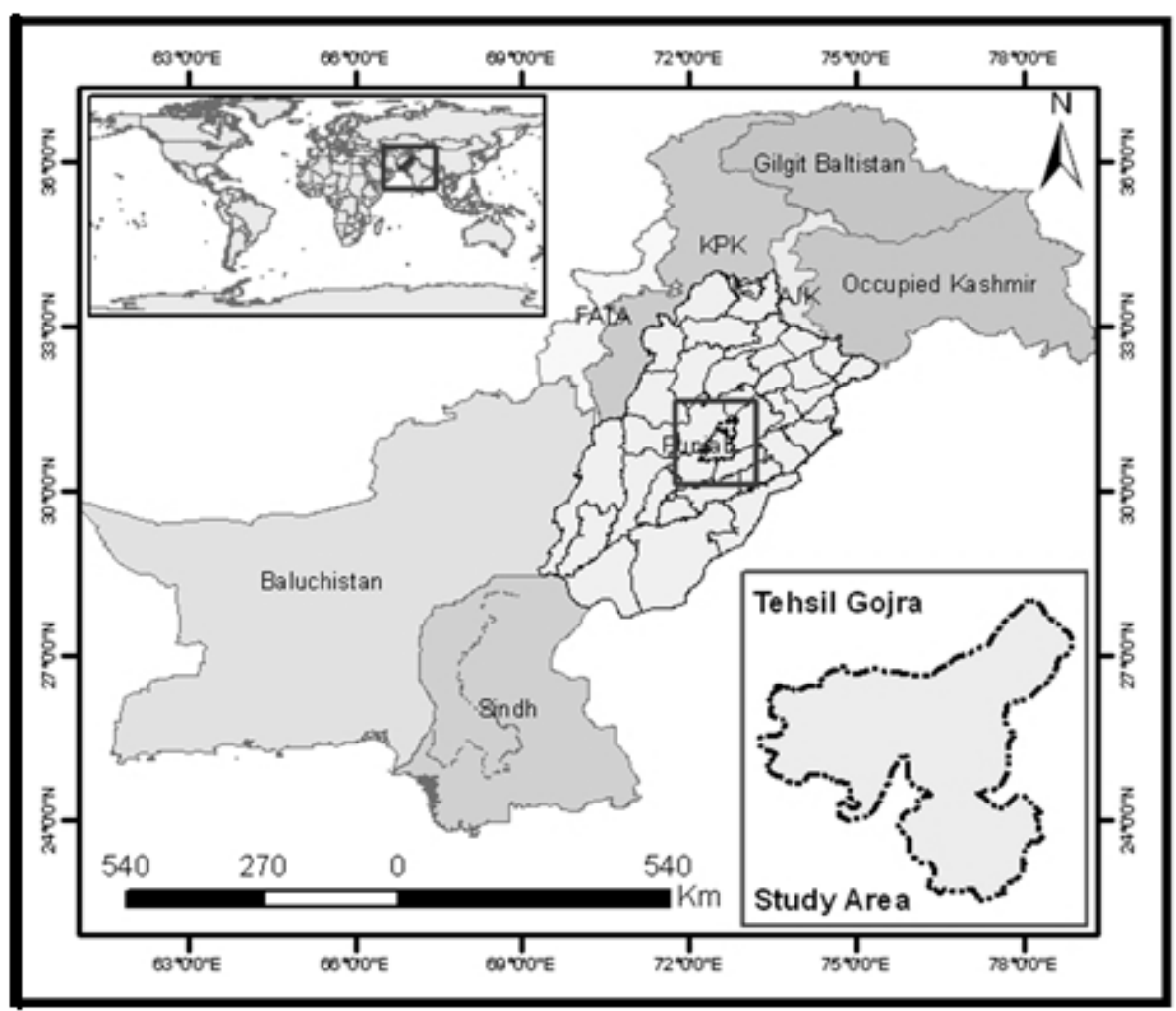

Map reference: Minallah et al. (2016)

Fig. 1. Map of the study area

\section{Data analysis}

Plant parts were arranged into various categories i.e. root, shoot, leaf and flower and data was analyzed using following formulae:

\section{Use Value (UV)}

The Use Value (UV) was determined to find relative importance of given species collected from the study area. It was calculated by following formula:

$$
U V=\Sigma U / N
$$

In UV formula, U represents the number of citation per specific plant and $\mathrm{N}$ represents the number of local informants.

\section{Relative Frequency of Citation (RFC)}

RFC was calculated by the given formula:

$$
\mathrm{RFC}=\frac{\mathrm{FC}}{\mathrm{N}}
$$

In this formula, FC was the number of informants for the use of that species and the $\mathrm{N}$ represents the total number of informants in the survey.

\section{Informant Consensus Factor (ICF)}

Information of related homogeneity mixture of disease category collected from informants was calculated by following formula:

$$
\mathrm{ICF}=\frac{\mathrm{Nur}-\mathrm{Nt}}{\mathrm{Nur}-1}
$$

Where, Nur describes the number of use citations from informants for a particular plant-use category and $\mathrm{Nt}$ represents the number of species or taxa utilized by all the informants for that specific plant use category. ICF had range between 0 to 1 , where ICF value 1 indicated highest level of informant consent and 0 was the lowest value

\section{Fidelity Level (FL)}

Fidelity level was used to search out the important advance species related to medicines.

$$
\mathrm{FL}(\%)=\frac{\mathrm{Np}}{\mathrm{N}} \times 100
$$

Where, $\mathrm{Np}$ for the number of species that is present in specific category. For accurate sum consumption for particular species symbol $\mathrm{N}$ was used. 


\section{Results}

During the present study related to ethnomedicinal values of the wild flora, 100 local inhabitants were interviewed, and information was recorded through questionnaire.

\section{Identification of plant families}

There were only 47 important wild ethnomedicinal plant species belonging to 20 plant families in the study area. There were more than 100 plant species including crops and wild plants but only 47 species were documented that were ethnomedicinally important among local community. The families were, Fabaceae, Asteraceae, Poaceae, Amaranthaceae, Moraceae, Euphorbiaceae, Solanaceae, Brassicaceae, Myrtaceae, Convolvulaceae, Malvaceae, Polygonaceae, Oxalidaceae, Apocynaceae, Cyperaceae, Papaveraceae, Portulacaceae, Primulaceae, Zygophyllaceae and Aizoaceae (Figure 2). Families were ranked based upon the number of the species present in the study area. Family Fabaceae was ranked at top with 9 species, Asteraceae and Poaceae with 5 species. There were 4 species of Amaranthaceae and Moraceae and 3 species were belonging to Euphorbiaceae and Solanaceae. All the other families identified in the area had only one species.

\section{Parts used in ethnomedicine}

Various parts of the plants i.e. roots, stem, leaves, latex, seeds and whole aerial parts were used to prepare different formulation for medicinal purposes. These parts were used fresh as well dried in different medicines. Drugs were prepared from these plant parts in the form of infusion, extraction, paste powder, decoction, and herbal tea. It was noted that $54 \%$ leaves, $32 \%$ fruits, $27 \%$ seeds, $31 \%$ whole plant, $18 \%$ roots, $10 \%$ stem and $2 \%$ latex about $1 \%$ were used in various prescriptions (Figure 3 ).

\section{Ethnomedicinal uses}

Information related to botanical name, family, UV, RFC, uses, part used, prescription, dosage, form of use and diseases are described in Table 1. It was noted from local informants that all the plants growing in the area have significance in traditional medicines to cure various diseases. Based upon the UV data, most commonly used plants at various locations in the study area were, A. modesta, $A$. aspera, C. album, C. intybus, D. sissoo, E. globulus and $R$. communis.

According to the data, $A$. modesta was a common tree in the area that had $0.73 \mathrm{UV}$ and $0.075 \mathrm{RFC}$ values. Its bark and gum mixed with honey are used in teeth problems, gastric problems. A. aspera was used to cure fever, respiratory problem and cough. Its leaves are mixed with honey and black pepper. Plant showed 0.85 UV and 0.088 RFC values.

For stomach, gastric and urinary disorders, people are using $C$. album with $0.78 \mathrm{UV}$ and $0.156 \mathrm{RFC}$ values. $C$. intybus has much importance in summer season as it is commonly used to reduce thirst in hot weather conditions. Its leaves are mixed with sugar to make summer drinks. Its herbal tea is also famous to improve digestion. Its UV was 0.74 and RFC was 0.132 . D. sissoo was mostly planted tree in the area for shade as well as medicinal importance. Its leaves, fruit and bark are used to control nosebleed, gonorrhea, stop vomiting and skin disorder. It has 0.72 UV and 0.075 RFC values. It was noted that $E$. globulus was used to treat common cold, respiratory infections, asthma and antiseptic. Its leaves are used to provide steam and oil is also applied to the asthma patients. It showed $0.76 \mathrm{UV}$ and $0.08 \mathrm{RFC}$ values.

$R$. communis was a common weed in the area. Its seeds and leaves are useful to treat rheumatic pain, joint pain and constipation with 0.74 UV and 0.19 RFC values (Table 1).

\section{Pharmacological data analysis}

Data for Informant Consensus Factor (ICF) by disease category and Fidelity Level (FL) value for most reported medicinal plants are given in Tables 2 and 3. According to respondent and ICF calculated values, these plants were recommended in many diseases include respiratory diseases, gastric problems, hepatitis, cardiovascular disorders and urogenital problem, kidney and liver disorders, diarrhea, constipation, fever, cold, cough, asthma, flue, bronchitis, vomiting, intestinal problems, arthritis, headache, hypertension, afterbirth problems, gallbladder problems, boils, gynecological disorders, malaria, cancer, paralysis, tumor, allergies, prolapse uterus, male infertility, sexual disorders, piles, snake bite, dropsy, cholera, toothache, rheumatism, stomach problems, gastric problems, intestinal problem, digestive problems, insect bite, body pain, epilepsy, convulsion, infections, nervous disorders, swellings, pneumonia, eye problems, bone pain, joint swellings, jaundice and diabetes (Table 2).

Fidelity Level (FL) value of most reported medicinal plants have been described in Table 3. A. modesta showed the highest FL value that was $87 \%$ that had used in teeth problems and gastric problems. Similarly, $82 \% \mathrm{FL}$ value was noted for $C$. album recommend for gastric problems 


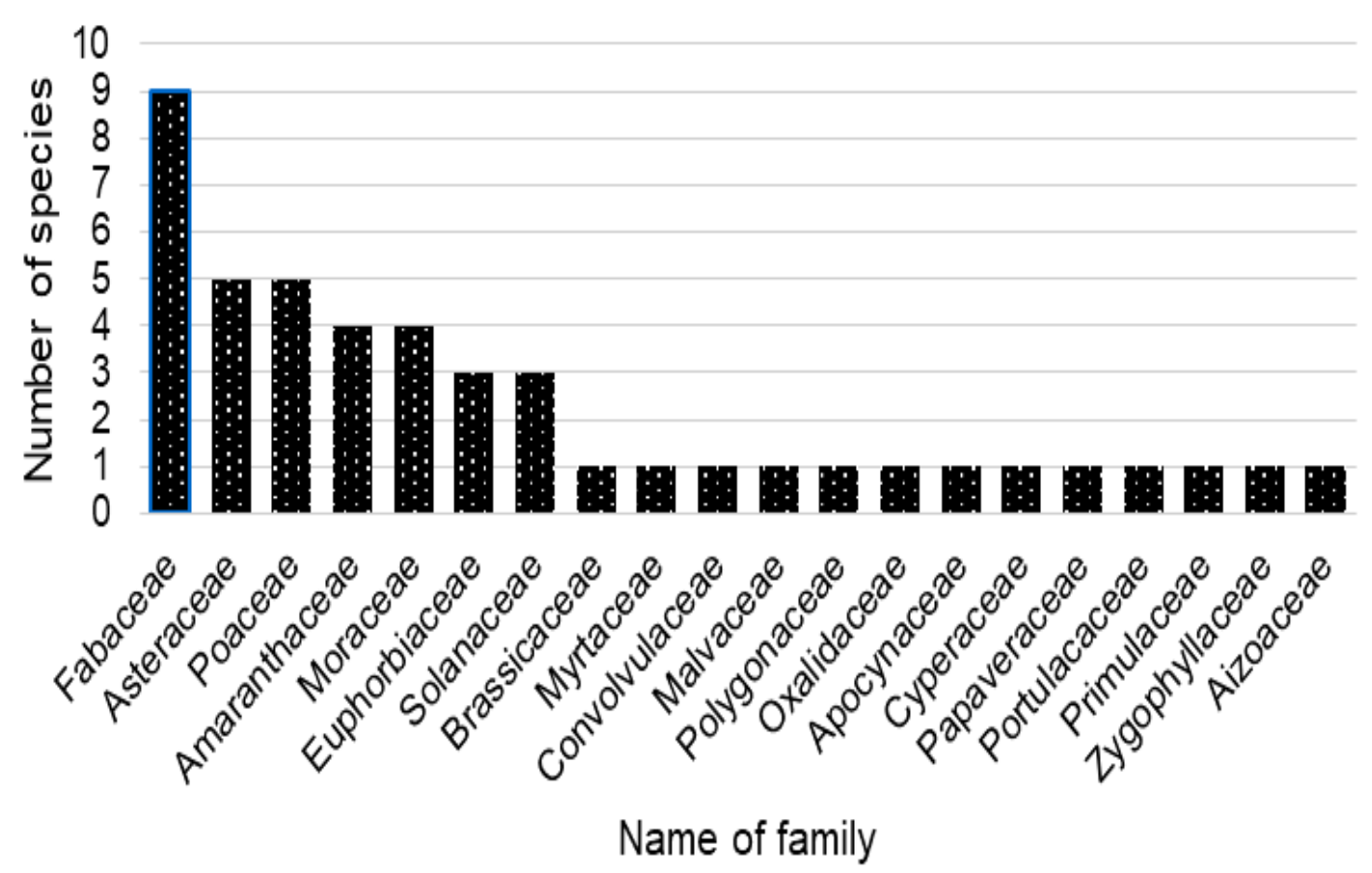

Figure 2. Name of family index with highest number of species used in the study area

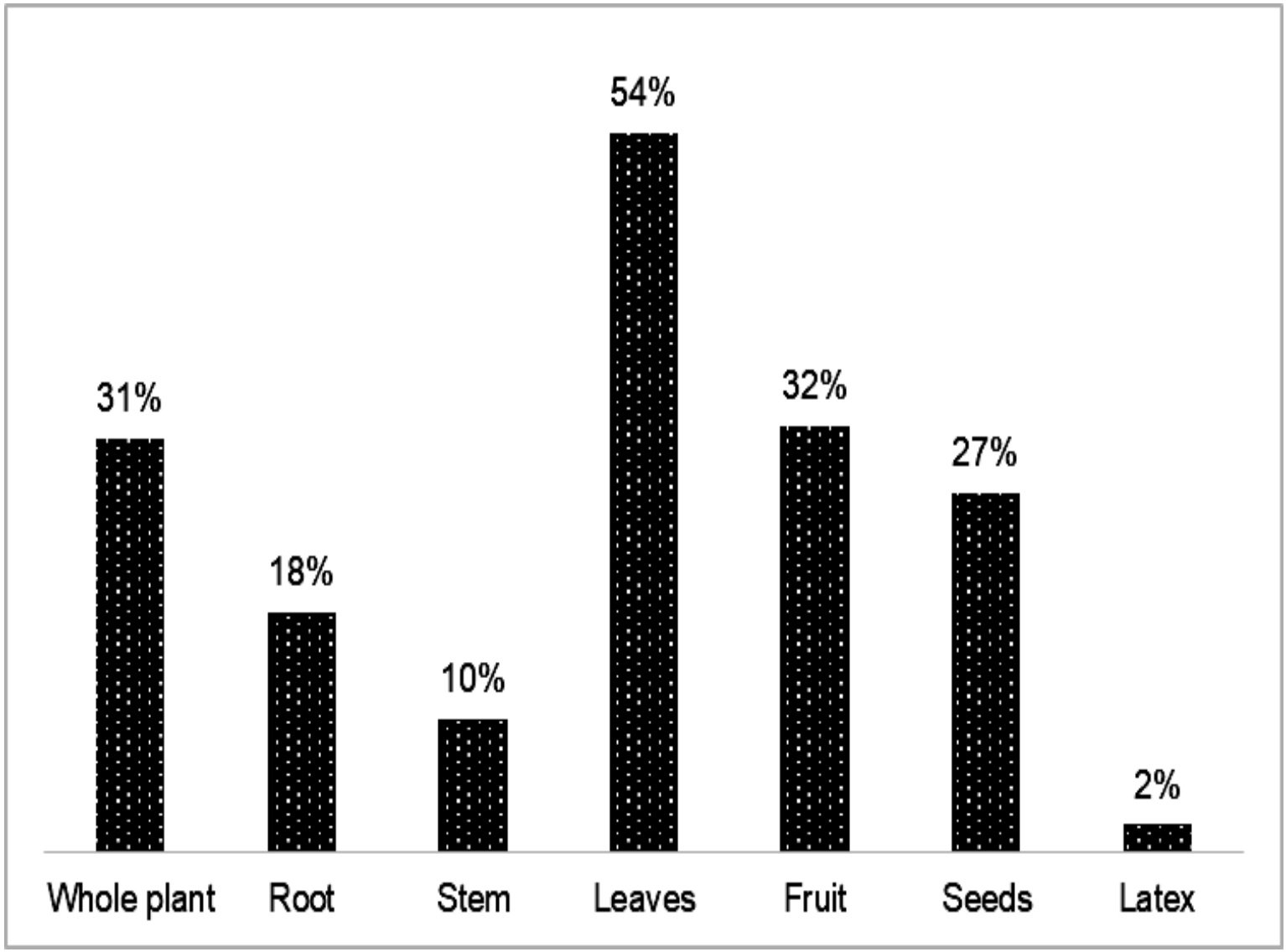

Figure 3. Percentage of plant parts used for medicinal purpose 
Table 1. Description of plants with uses, method and prescription

\begin{tabular}{|c|c|c|c|c|c|c|c|c|c|}
\hline Botanical Name & Family & $\begin{array}{l}\text { Voucher } \\
\text { no. }\end{array}$ & $\begin{array}{l}\text { Use } \\
\text { value } \\
\text { (UV) }\end{array}$ & $\begin{array}{l}\text { Relative } \\
\text { Frequency } \\
\text { of Citation } \\
\text { (RFC) }\end{array}$ & Uses & Prescription & Application & $\begin{array}{l}\text { Time of } \\
\text { use }\end{array}$ & $\begin{array}{l}\text { Form of } \\
\text { use }\end{array}$ \\
\hline $\begin{array}{l}\text { Trianthema } \\
\text { portulacastrum } \mathrm{L} .\end{array}$ & Aizoaceae & Aiz-03/20 & 0.54 & 0.16 & $\begin{array}{l}\text { Useful for the } \\
\text { treatment of } \\
\text { constipation, asthma }\end{array}$ & $\begin{array}{l}\text { Plant juice is } \\
\text { extracted }\end{array}$ & Oral & $\begin{array}{l}3 \text { times } \\
\text { per day }\end{array}$ & Fresh \\
\hline Achyranthes aspera L. & Amaranthaceae & Ama-17/20 & 0.85 & 0.088 & $\begin{array}{l}\text { Fever, respiratory } \\
\text { problems, cough }\end{array}$ & $\begin{array}{l}\text { Fresh/dry } \\
\text { leaves mixed } \\
\text { with honey and } \\
\text { black pepper }\end{array}$ & Oral & $\begin{array}{l}3 \text { times } \\
\text { per day }\end{array}$ & $\begin{array}{l}\text { Fresh/dry } \\
\text { leaves }\end{array}$ \\
\hline Amaranthus viridis $\mathrm{L}$. & Amaranthaceae & Ama-18/20 & 0.32 & 0.043 & Insect bite, malaria & $\begin{array}{l}\text { Seeds are } \\
\text { mixed with rice } \\
\text { or honey }\end{array}$ & Oral & $\begin{array}{l}2 \text { times } \\
\text { per day }\end{array}$ & $\begin{array}{l}\text { Seeds } \\
\text { (dry) }\end{array}$ \\
\hline Chenopodium album $\mathrm{L}$. & Amaranthaceae & Ama-14/19 & 0.78 & 0.156 & $\begin{array}{l}\text { Stomach/gastric, and } \\
\text { urinary disorders }\end{array}$ & $\begin{array}{l}\text { Leaves mixed } \\
\text { with water for } \\
\text { kidney } \\
\text { disorder(herbal } \\
\text { tea) }\end{array}$ & Oral & $\begin{array}{l}2 \text { times } \\
\text { per day }\end{array}$ & Fresh \\
\hline $\begin{array}{l}\text { Chenopodium murale } \\
\text { (L.) S. Fuentes, Uotila \& } \\
\text { Borsch }\end{array}$ & Amaranthaceae & Ama-15/19 & 0.61 & 0.054 & $\begin{array}{l}\text { Dry cough, pulmonary } \\
\text { obstruction }\end{array}$ & $\begin{array}{l}\text { Whole plant } \\
\text { part are } \\
\text { recommended } \\
\text { to rub at chest }\end{array}$ & External & $\begin{array}{l}2 \text { times } \\
\text { per day }\end{array}$ & $\begin{array}{l}\text { Dry and } \\
\text { fresh }\end{array}$ \\
\hline $\begin{array}{l}\text { Calotropis procera } \\
\text { (Aiton) Dryand. }\end{array}$ & Apocynaceae & Apo-04/19 & 0.66 & 0.16 & Snake bite, body pain & $\begin{array}{l}\text { Mixed leaves } \\
\text { with mustard } \\
\text { oil and rubbed }\end{array}$ & External & $\begin{array}{l}3-4 \\
\text { times } \\
\text { per day }\end{array}$ & Dry \\
\hline Cichorium intybus L. & Asteraceae & Ast-33/19 & 0.74 & 0.132 & $\begin{array}{l}\text { Reduce thirst, } \\
\text { improve digestion and } \\
\text { liver problem }\end{array}$ & $\begin{array}{l}\text { Fresh/dry } \\
\text { leaves to make } \\
\text { herbal tea to } \\
\text { improve } \\
\text { digestion as } \\
\text { well as mixed } \\
\text { with sugar to } \\
\text { make summer } \\
\text { drinks }\end{array}$ & Oral & $\begin{array}{l}2 \text { times } \\
\text { per day }\end{array}$ & Fresh/ dry \\
\hline $\begin{array}{l}\text { Cirsium arvense (L.) } \\
\text { Scop. }\end{array}$ & Asteraceae & Ast-34/19 & 0.43 & 0.14 & $\begin{array}{l}\text { Improves digestion } \\
\text { and used for child } \\
\text { worms }\end{array}$ & $\begin{array}{l}\text { Decoction of } \\
\text { roots }\end{array}$ & Oral & $\begin{array}{l}3 \text { times } \\
\text { per day }\end{array}$ & Dry \\
\hline Eclipta prostrata (L.) L. & Asteraceae & Ast-36/19 & 0.32 & 0.12 & $\begin{array}{l}\text { Useful for curing } \\
\text { snake bite. Anti- }\end{array}$ & $\begin{array}{l}\text { Whole plant is } \\
\text { mixed with } \\
\text { olive oil }\end{array}$ & Oral & $\begin{array}{l}1 \text { time } \\
\text { per day }\end{array}$ & Fresh \\
\hline
\end{tabular}




\begin{tabular}{|c|c|c|c|c|c|c|c|c|c|}
\hline & & & & & $\begin{array}{l}\text { bacterial and anti- } \\
\text { oxidant. }\end{array}$ & & & & \\
\hline Sonchus asper (L.) Hill & Asteraceae & Ast-43/20 & 0.34 & 0.014 & $\begin{array}{l}\text { Antimicrobial activity, } \\
\text { paste is useful to cure } \\
\text { wounds }\end{array}$ & $\begin{array}{l}\text { Seeds is } \\
\text { soaked in } 1 \\
\text { cup water for } \\
\text { one night }\end{array}$ & External & $\begin{array}{l}1 \text { time } \\
\text { per day }\end{array}$ & Fresh \\
\hline Xanthium strumarium L. & Asteraceae & Ast-43/19 & 0.54 & 0.18 & $\begin{array}{l}\text { Anti-inflammatory, } \\
\text { antioxidant and } \\
\text { antibacterial } \\
\text { properties }\end{array}$ & $\begin{array}{l}\text { Leaves are } \\
\text { used to make } \\
\text { paste }\end{array}$ & External & $\begin{array}{l}1 \text { time } \\
\text { per day }\end{array}$ & Fresh \\
\hline Lepidium didymium L. & Brassicaceae & Bra-16/20 & 0.52 & 0.19 & $\begin{array}{l}\text { Skin diseases and } \\
\text { diabetes, useful for } \\
\text { the treatment of } \\
\text { gastrointestinal ulcers }\end{array}$ & $\begin{array}{l}\text { Poultice of the } \\
\text { leaves is } \\
\text { applied }\end{array}$ & External & $\begin{array}{l}2 \text { times } \\
\text { per day }\end{array}$ & Dry \\
\hline Convolvulus arvensis L. & Convolvulaceae & Con-03/19 & 0.48 & 0.06 & $\begin{array}{l}\text { Cure urinary tract } \\
\text { infections and ulcers. }\end{array}$ & $\begin{array}{l}\text { Make juice of } \\
\text { roots }\end{array}$ & Oral & $\begin{array}{l}\text { Twice a } \\
\text { day }\end{array}$ & Dry \\
\hline Cyperus rotundus L. & Cyperaceae & Сур-04/20 & 0.23 & 0.08 & $\begin{array}{l}\text { Anti-diabetic, anti- } \\
\text { inflammatory and } \\
\text { antimalarial } \\
\text { properties. }\end{array}$ & $\begin{array}{l}\text { Powder of } \\
\text { rhizome }\end{array}$ & Oral & $\begin{array}{l}1 \text { time } \\
\text { per day }\end{array}$ & Dry \\
\hline Euphorbia helioscopia L. & Euphorbiaceae & Eup-18/20 & 0.53 & 0.11 & $\begin{array}{l}\text { Used in constipation, } \\
\text { athlete's foot and } \\
\text { intestinal problems. }\end{array}$ & $\begin{array}{l}\text { Take its leaves } \\
\text { as herbal tea }\end{array}$ & Oral & $\begin{array}{l}3 \text { times } \\
\text { per day }\end{array}$ & Fresh \\
\hline Euphorbia prostrata Aiton & Euphorbiaceae & Eup-15/20 & 0.59 & 0.14 & $\begin{array}{l}\text { Useful for curing } \\
\text { jaundice, fever and } \\
\text { kill intestinal worms. } \\
\text { Act as anti- } \\
\text { inflammatory and } \\
\text { anti-bacterial. }\end{array}$ & $\begin{array}{l}\text { Plant part that } \\
\text { grows above- } \\
\text { ground use to } \\
\text { make medicine }\end{array}$ & Oral & $\begin{array}{l}2 \text { times } \\
\text { per day }\end{array}$ & Fresh \\
\hline Ricinus communis $\mathrm{L}$. & Euphorbiaceae & Eup-21/19 & 0.74 & 0.19 & $\begin{array}{l}\text { Useful for curing } \\
\text { rheumatic pain, joint } \\
\text { pain and constipation }\end{array}$ & $\begin{array}{l}\text { Seeds and } \\
\text { leaves are } \\
\text { used to make } \\
\text { paste }\end{array}$ & $\begin{array}{l}\text { External and } \\
\text { Oral }\end{array}$ & $\begin{array}{l}2 \text { times } \\
\text { per day }\end{array}$ & Dry \\
\hline Acacia modesta Wall. & Fabaceae & Fab-16/20 & 0.73 & 0.075 & $\begin{array}{l}\text { Teeth problems, } \\
\text { gastric problems }\end{array}$ & $\begin{array}{l}\text { Bark and gum } \\
\text { mixed with } \\
\text { honey }\end{array}$ & Oral & $\begin{array}{l}2 \text { times } \\
\text { per day }\end{array}$ & Dry \\
\hline Acacia nilotica (L.) Delile. & Fabaceae & Fab-18/20 & 0.66 & 0.055 & $\begin{array}{l}\text { Pyrexia, digestive } \\
\text { problems, } \\
\text { gonorrhoea, } \\
\text { antiseptic, skin } \\
\text { infections and blood } \\
\text { purification }\end{array}$ & $\begin{array}{l}\text { Flower, bark, } \\
\text { seeds, gum } \\
\text { twinges }\end{array}$ & Oral & $\begin{array}{l}2 \text { times } \\
\text { per day }\end{array}$ & Fresh/dry \\
\hline
\end{tabular}




\begin{tabular}{|c|c|c|c|c|c|c|c|c|c|}
\hline Albizia lebbeck (L.) Benth & Fabaceae & Fab-17/20 & 0.55 & 0.043 & $\begin{array}{l}\text { Lung problems, blood } \\
\text { purification abdominal } \\
\text { tumours, boils, cough, } \\
\text { hernia }\end{array}$ & $\begin{array}{l}\text { Stem, bark or } \\
\text { leaves as } \\
\text { herbal tea }\end{array}$ & Oral & $\begin{array}{l}1 \text { time } \\
\text { per day }\end{array}$ & Fresh \\
\hline Dalbergia sissoo DC. & Fabaceae & Fab-22/19 & 0.72 & 0.075 & $\begin{array}{l}\text { Controls nose bleed, } \\
\text { gonorrhoea, stop } \\
\text { vomiting and skin } \\
\text { disorder }\end{array}$ & $\begin{array}{l}\text { Take } 2 \mathrm{~g} \text { leaves } \\
\text { and fruit and } \\
\text { boil it in water }\end{array}$ & Oral & $\begin{array}{l}2 \text { times } \\
\text { per day }\end{array}$ & Fresh \\
\hline Lathyrus aphaca L. & Fabaceae & Fab-24/19 & 0.23 & 0.03 & $\begin{array}{l}\text { Useful to cure snake } \\
\text { bite. }\end{array}$ & $\begin{array}{l}\text { Take } 2 \text { spoon } \\
\text { of seed and } \\
\text { mix with water }\end{array}$ & Oral & $\begin{array}{l}1 \text { time } \\
\text { per day }\end{array}$ & Dry \\
\hline Medicago polymorpha L. & Fabaceae & Fab-25/19 & 0.62 & 0.13 & $\begin{array}{l}\text { Used to cure kidney, } \\
\text { intestinal and bladder } \\
\text { infections }\end{array}$ & $\begin{array}{l}\text { Powder of } \\
\text { seeds mixed } \\
\text { with water to } \\
\text { make a mush }\end{array}$ & Oral & $\begin{array}{l}2 \text { times } \\
\text { per day }\end{array}$ & Dry \\
\hline Medicago sativa L. & Fabaceae & Fab-35/19 & 0.56 & 0.22 & $\begin{array}{l}\text { Body tonic. Enhance } \\
\text { metabolism and } \\
\text { increase milk } \\
\text { production in } \\
\text { livestock }\end{array}$ & $\begin{array}{l}\text { Whole plant } \\
\text { mixed with oat, } \\
\text { alfalfa to make } \\
\text { syrup }\end{array}$ & Oral & $\begin{array}{l}2 \text { times } \\
\text { per day }\end{array}$ & Fresh \\
\hline Melilotus indicus (L.) All. & Fabaceae & Fab-25/20 & 0.56 & 0.34 & $\begin{array}{l}\text { Useful in diarrhea and } \\
\text { bowl complaints, } \\
\text { intestinal problems. }\end{array}$ & $\begin{array}{l}\text { Seed or whole } \\
\text { plant is used } \\
\text { to make tablets } \\
\text { or dry powder }\end{array}$ & Oral & $\begin{array}{l}1 \text { time } \\
\text { per day }\end{array}$ & Dry \\
\hline Vicia sativa $\mathrm{L}$. & Fabaceae & Fab-37/19 & 0.45 & 0.14 & $\begin{array}{l}\text { Useful as emollient } \\
\text { and have anti- } \\
\text { inflammatory and } \\
\text { antioxidant properties }\end{array}$ & $\begin{array}{l}\text { Poultice is } \\
\text { recommended } \\
\text { for skin } \\
\text { infection }\end{array}$ & External & $\begin{array}{l}1 \text { time } \\
\text { per day }\end{array}$ & Dry \\
\hline Malva parviflora L. & Malvaceae & Mal-19/19 & 0.28 & 0.12 & $\begin{array}{l}\text { Antimicrobial, } \\
\text { antibacterial and anti- } \\
\text { inflammatory } \\
\text { properties }\end{array}$ & $\begin{array}{l}\text { Leaf is boiled } \\
\text { in } 3-4 \text { cup } \\
\text { water mixed } \\
\text { with cow milk }\end{array}$ & Oral & $\begin{array}{l}\text { Daily in } \\
\text { the } \\
\text { evening }\end{array}$ & Fresh \\
\hline Ficus benghalensis L. & Moraceae & Mor-11/20 & 0.62 & 0.031 & $\begin{array}{l}\text { Cough, flu, Chronic } \\
\text { flu \& influenza, } \\
\text { gonorrhoea, } \\
\text { impotencey }\end{array}$ & $\begin{array}{l}\text { Latex, twings. } \\
\text { Latex } 20 \mathrm{~g}, \\
\text { milk } 10 \mathrm{~g}, \mathrm{gum} \\
\text { of Acacia } 20 \mathrm{~g} \\
\text { and Asparagus } \\
20 \mathrm{~g} \text { and make } \\
\text { tablets }\end{array}$ & Oral & $\begin{array}{l}2 \text { times } \\
\text { per day }\end{array}$ & $\begin{array}{l}\text { Fresh or } \\
\text { dry }\end{array}$ \\
\hline Ficus religiosa L. & Moraceae & Mor-12/20 & 0.54 & 0.042 & $\begin{array}{l}\text { Nervous problems, } \\
\text { gonorrhea }\end{array}$ & $\begin{array}{l}\text { Milky latex } \\
\text { mixed with } \\
\text { honey }\end{array}$ & Oral & $\begin{array}{l}1 \text { time } \\
\text { per day }\end{array}$ & Dry \\
\hline
\end{tabular}




\begin{tabular}{|c|c|c|c|c|c|c|c|c|c|}
\hline Morus alba L. & Moraceae & Mor-04/19 & 0.43 & 0.08 & $\begin{array}{l}\text { Useful for treatment } \\
\text { of hepatitis, liver } \\
\text { diseases }\end{array}$ & $\begin{array}{l}\text { Fruit, root and } \\
\text { leaves of are } \\
\text { mixed with } \\
\text { honey }\end{array}$ & Oral & $\begin{array}{l}1 \text { time } \\
\text { per day }\end{array}$ & Fresh/ dry \\
\hline Morus nigra L. & Moraceae & Mor-05/19 & 0.56 & 0.32 & $\begin{array}{l}\text { Used for sore throat, } \\
\text { respiratory infection }\end{array}$ & $\begin{array}{l}\text { Fruit mixed with } \\
\text { sugar and } \\
\text { water to make } \\
\text { syrup }\end{array}$ & Oral & $\begin{array}{l}2 \text { times } \\
\text { per day }\end{array}$ & Fresh \\
\hline $\begin{array}{l}\text { Eucalyptus globulus } \\
\text { Labill. }\end{array}$ & Myrtaceae & Myr-03/20 & 0.76 & 0.08 & $\begin{array}{l}\text { Common cold, } \\
\text { respiratory infections, } \\
\text { asthma and antiseptic }\end{array}$ & $\begin{array}{l}\text { Leaves, oil is } \\
\text { mixed in water } \\
\text { for stream }\end{array}$ & Oral & $\begin{array}{l}1 \text { time } \\
\text { par day }\end{array}$ & $\begin{array}{l}\text { Dry/fresh } \\
\text { leaves } \\
\text { and oil }\end{array}$ \\
\hline Oxalis corniculata L. & Oxalidaceae & Oxa-05/19 & 0.56 & 0.17 & $\begin{array}{l}\text { Useful in redness of } \\
\text { eye and scurvy, and } \\
\text { anti-microbial activity. } \\
\text { Useful to cure gastric } \\
\text { troubles. }\end{array}$ & $\begin{array}{l}\text { Fresh or dry } \\
\text { leaves are } \\
\text { used to make } \\
\text { powder }\end{array}$ & Oral & $\begin{array}{l}2 \text { times } \\
\text { per day }\end{array}$ & $\begin{array}{l}\text { Fresh } \\
\text { plus dry }\end{array}$ \\
\hline $\begin{array}{l}\text { Fumaria indica } \\
\text { (Hausskn.) Pugsley }\end{array}$ & Papaveraceae & Pap-06/20 & 0.69 & 0.043 & $\begin{array}{l}\text { Used in fever, liver } \\
\text { problems and } \\
\text { diarrhea }\end{array}$ & $\begin{array}{l}\text { Make } \\
\text { decoction of } \\
\text { leaves with } \\
\text { water }\end{array}$ & Oral & $\begin{array}{l}2 \text { times } \\
\text { per day }\end{array}$ & $\begin{array}{l}\text { Fresh and } \\
\text { dry }\end{array}$ \\
\hline Avena sativa $\mathrm{L}$. & Poaceae & Poa-29/20 & 0.54 & 0.081 & $\begin{array}{l}\text { Stomach problems, } \\
\text { diabetes }\end{array}$ & $\begin{array}{l}\text { Seeds grind } \\
\text { with sugar }\end{array}$ & Oral & $\begin{array}{l}\text { I time } \\
\text { per day }\end{array}$ & $\begin{array}{l}\text { Seeds } \\
\text { (dry) }\end{array}$ \\
\hline $\begin{array}{l}\text { Cynodon dactylon (L.) } \\
\text { Pers. }\end{array}$ & Poaceae & Poa-29/19 & 0.46 & 0.11 & $\begin{array}{l}\text { Blood purifier and } \\
\text { used in runny nose } \\
\text { due to flu }\end{array}$ & $\begin{array}{l}\text { Whole plant } \\
\text { paste }\end{array}$ & $\begin{array}{l}\text { Oral and } \\
\text { External }\end{array}$ & $\begin{array}{l}2 \text { to } 3 \\
\text { times } \\
\text { per day }\end{array}$ & Fresh/dry \\
\hline $\begin{array}{l}\text { Digitaria bicornis (Lam.) } \\
\text { Roem. \& Schult. }\end{array}$ & Poaceae & Poa-42/19 & 0.31 & 0.23 & Useful to kill microbes & $\begin{array}{l}\text { Leaves and } \\
\text { grinded seeds } \\
\text { to make } \\
\text { poultice }\end{array}$ & External & $\begin{array}{l}1 \text { time } \\
\text { per day }\end{array}$ & $\begin{array}{l}\text { Dry and } \\
\text { fresh }\end{array}$ \\
\hline Phalaris minor Retz. & Poaceae & Poa-44/20 & 0.61 & 0.18 & $\begin{array}{l}\text { Cure cough, cold, } \\
\text { asthma and } \\
\text { dysentery }\end{array}$ & $\begin{array}{l}\text { Dry leaves are } \\
\text { used to make } \\
\text { powder }\end{array}$ & Oral & $\begin{array}{l}1 \text { time } \\
\text { per day }\end{array}$ & Fresh \\
\hline Saccharum munja Roxb. & Poaceae & Poa-33/20 & 0.23 & 0.09 & $\begin{array}{l}\text { Used during baby } \\
\text { birth to control pains }\end{array}$ & $\begin{array}{l}\text { Whole plant is } \\
\text { boiled in water }\end{array}$ & External & $\begin{array}{l}\text { Morning } \\
\text { time }\end{array}$ & Fresh \\
\hline Rumex dentatus L. & Polygonaceae & Pol-12/19 & 0.61 & 0.21 & $\begin{array}{l}\text { Antioxidant, anti- } \\
\text { inflammatory and } \\
\text { antimicrobial activity. } \\
\text { Act as appetizer, cure } \\
\text { constipation and } \\
\text { diarrhea }\end{array}$ & $\begin{array}{l}\text { Take its leaves } \\
\text { grind them and } \\
\text { make its juice }\end{array}$ & External & $\begin{array}{l}2 \text { times } \\
\text { per day }\end{array}$ & Dry \\
\hline
\end{tabular}




\begin{tabular}{|c|c|c|c|c|c|c|c|c|c|}
\hline Portulaca oleracea L. & Portulacaceae & Por-04/20 & 0.63 & 0.27 & $\begin{array}{l}\text { Recommended in } \\
\text { fever, ulcer and } \\
\text { abnormal uterine } \\
\text { bleeding }\end{array}$ & $\begin{array}{l}\text { Whole plant is } \\
\text { used to make } \\
\text { poultice }\end{array}$ & Oral & $\begin{array}{l}1 \text { time } \\
\text { per day }\end{array}$ & $\begin{array}{l}\text { Dry or } \\
\text { fresh }\end{array}$ \\
\hline Anagallis arvensis $\mathrm{L}$. & Primulaceae & Pri-03/20 & 0.34 & 0.091 & $\begin{array}{l}\text { Wound healings, skin } \\
\text { infections }\end{array}$ & $\begin{array}{l}\text { Fresh leaves } \\
\text { to make } \\
\text { poultice }\end{array}$ & External & $\begin{array}{l}2 \text { time } \\
\text { per day }\end{array}$ & $\begin{array}{l}\text { Fresh as } \\
\text { well as } \\
\text { dry }\end{array}$ \\
\hline Datura metel L. & Solanaceae & Sol-11/18 & 0.42 & 0.19 & $\begin{array}{l}\text { Curing paralyzed } \\
\text { portion of the body. } \\
\text { Relieve snake bite } \\
\text { Cure arthritis and } \\
\text { have antimicrobial } \\
\text { properties }\end{array}$ & $\begin{array}{l}\text { Take its leaves } \\
\text { to make paste }\end{array}$ & External & $\begin{array}{l}2 \text { times } \\
\text { per day }\end{array}$ & Fresh \\
\hline Solanum nigrum L. & Solanaceae & Sol-14/19 & 0.62 & 0.13 & $\begin{array}{l}\text { Prevent respiratory, } \\
\text { hepatic and stomach } \\
\text { problems. Cure eye } \\
\text { problems, fever, piles } \\
\text { and diabetes. }\end{array}$ & $\begin{array}{l}\text { Leaves mixed } \\
\text { with honey and } \\
\text { other herbs }\end{array}$ & Oral & $\begin{array}{l}2 \text { times } \\
\text { per day }\end{array}$ & Dry \\
\hline $\begin{array}{l}\text { Withania somnifera (L.) } \\
\text { Dunal }\end{array}$ & Solanaceae & Sol-19/20 & 0.51 & 0.21 & $\begin{array}{l}\text { Cure neurological } \\
\text { disorders and men } \\
\text { sexual health }\end{array}$ & $\begin{array}{l}\text { Roots are used } \\
\text { to make herbal } \\
\text { tea with milk }\end{array}$ & Oral & $\begin{array}{l}\text { time } \\
\text { per day }\end{array}$ & Dry \\
\hline Tribulus terrestris $\mathrm{L}$. & Zygophyllaceae & Zyg-02/18 & 0.68 & 0.23 & $\begin{array}{l}\text { Used in impotency, } \\
\text { men sexual power, } \\
\text { body tonic, useful for } \\
\text { the treatment of } \\
\text { kidney stones and } \\
\text { urinary tract infections }\end{array}$ & $\begin{array}{l}\text { Extract of } \\
\text { leaves is used } \\
\text { and powder of } \\
\text { seeds }\end{array}$ & Oral & $\begin{array}{l}1 \text { time } \\
\text { per day }\end{array}$ & Fresh \\
\hline
\end{tabular}


Table 2. Informant Consensus Factor (ICF) by disease category reported in study area

\begin{tabular}{|c|c|c|c|c|c|}
\hline Disease Category & $\begin{array}{l}\text { No. of Use } \\
\text { Reports } \\
\text { (Nur) }\end{array}$ & $\begin{array}{l}\text { No. of } \\
\text { Species } \\
\text { Used (Nt) }\end{array}$ & Nur-Nt & Nur-1 & ICF \\
\hline $\begin{array}{l}\text { Nervous disorders, Swellings, } \\
\text { Pneumonia, Eye problems }\end{array}$ & 15 & 9 & 6 & 14 & 0.42 \\
\hline Headache, Hypertension & 17 & 7 & 10 & 16 & 0.62 \\
\hline $\begin{array}{l}\text { Afterbirth problems, Gallbladder } \\
\text { problems, Boils, Gynecological } \\
\text { disorders }\end{array}$ & 19 & 8 & 11 & 18 & 0.61 \\
\hline $\begin{array}{l}\text { Prolapse uterus, Male infertility, } \\
\text { Sexual disorders }\end{array}$ & 19 & 5 & 14 & 18 & 0.77 \\
\hline $\begin{array}{l}\text { Insect bite, Body pain, Epilepsy, } \\
\text { Convulsion, Infections }\end{array}$ & 19 & 6 & 13 & 18 & 0.72 \\
\hline $\begin{array}{l}\text { Malaria, Cancer, Peralysis, } \\
\text { Tumor, Allergies }\end{array}$ & 22 & 12 & 10 & 21 & 0.47 \\
\hline $\begin{array}{l}\text { Respiratory diseases, Gastric } \\
\text { problems, Hepatitis }\end{array}$ & 24 & 10 & 14 & 23 & 0.61 \\
\hline $\begin{array}{l}\text { Piles, Snake bite, Dropsy', } \\
\text { Cholera, Toothache, } \\
\text { Rheumatism }\end{array}$ & 26 & 13 & 13 & 25 & 0.52 \\
\hline $\begin{array}{l}\text { Bone pain, Joint swellings, } \\
\text { Jaundice }\end{array}$ & 26 & 19 & 7 & 25 & 0.28 \\
\hline $\begin{array}{l}\text { Jaundice, Malaria, Bronchitis, } \\
\text { Vomiting, Intestinal problems, } \\
\text { Arthritis }\end{array}$ & 27 & 15 & 12 & 26 & 0.46 \\
\hline Diabetes & 27 & 13 & 14 & 26 & 0.53 \\
\hline $\begin{array}{l}\text { Cardiovascular disorders and } \\
\text { Urogenital problem }\end{array}$ & 30 & 14 & 16 & 29 & 0.55 \\
\hline Kidney and liver disorders & 31 & 13 & 18 & 30 & 0.6 \\
\hline Skin infections, inflammation & 87 & 52 & 35 & 86 & 0.40 \\
\hline $\begin{array}{l}\text { Stomach problems, Gastric } \\
\text { problems, Intestinal problem, } \\
\text { Digestive problems }\end{array}$ & 35 & 9 & 26 & 34 & 0.76 \\
\hline Loose motion, Constipation & 45 & 20 & 25 & 44 & 0.56 \\
\hline $\begin{array}{l}\text { Wound healing, Ulcer, Skin } \\
\text { diseases, Tonic }\end{array}$ & 65 & 38 & 27 & 64 & 0.42 \\
\hline $\begin{array}{l}\text { Fever, Cold, Cough, Asthma, } \\
\text { Flue }\end{array}$ & 67 & 37 & 30 & 66 & 0.45 \\
\hline
\end{tabular}

Where, Nur describes he number of use citations from inants for a particular plant-use category and Nt represents the number of species

Table 3. Fidelity Level (FL) value for most reported medicinal plants

\begin{tabular}{|c|c|c|c|}
\hline Scientific name & Common name & Major ailment & $\begin{array}{l}\text { Fidelity } \\
\text { level (FL) \% }\end{array}$ \\
\hline Acacia modesta Wall. & Phulahi & $\begin{array}{l}\text { Teeth problems, gastric } \\
\text { problems }\end{array}$ & 87 \\
\hline Achyranthes aspera L. & Putkanda & $\begin{array}{l}\text { Gastrointestinal disorders and } \\
\text { menstrual pain }\end{array}$ & 77 \\
\hline Amaranthus viridis $\mathrm{L}$. & Ghunar & Snake bite & 64 \\
\hline Anagallis arvensis L. & Bili booti & Wound healing & 76 \\
\hline Calotropis procera (Aiton) W.T.Aiton & Aak & Wound healing & 63 \\
\hline Chenopodium album L. & Bathu & Gastric problems & 82 \\
\hline Cichorium intybus L. & Kasani & Liver problem, blood purifier & 58 \\
\hline Cynodon dactylon (L.) Pers. & Khabbal, talla ghas & Inflammation & 38 \\
\hline Eclipta prostrata L. & Bhangra & Snake bite & 60 \\
\hline Fumaria indica (Hausskn.) Pugsley & Shahtra & Fever & 70 \\
\hline Rumex dentatus L. & Jangli palak & Constipation & 63 \\
\hline Solanum nigrum $\mathrm{L}$. & Kainch mainch & Stomach burn & 67 \\
\hline Trianthema portulacastrum L. & Itsit & Constipation & 76 \\
\hline Vicia sativa L. & Matri & Skin diseases & 43 \\
\hline
\end{tabular}




\section{Discussion}

Ethnobotanical surveys have much importance to collect the folk knowledge about the medicinal floras that are the basis of various medicines in the world (Ali et al. 2020). Ethnomedicinal flora of any area shows its significance among the local communities as well as it is the major source of raw materials for industries (Njoroge et al., 2004). Wild plants are mostly used as food as well as it is good source of medicines (Abbasi et al, 2016). Weeds of any area have much role in folk medicines. People rely on these wild plants (Rehman et al., 2020). During this study, it was noted that Tehsil Gojra has number of wild plants, which have medicinal significance. There are many wild plants in the area which were reported first time in this study i.e. Cirsium arvense, Digitaria bicornis, Lepidium didymium Withania somnifera. In literature, Muhammad et al. (2009) conducted the study of Tehsil Gojra too but they only described the weeds of wheat crop that had great significance to cure various diseases of Tehsil Gojra, Pakistan. Pakistan has different seasons and variety of soils that's why country is rich with medicinal plants. Many researchers reported the efficacy of those plants in various medicines as reported during this study as Rehman et al. (2020) reported 32 families of weeds and showed the significance of important seasonal weeds growing in district Gujrat, Pakistan against many diseases by local inhabitants.

Ethnomedicinal report of our area is in line with the findings of many other researches carried out in the same area and neighboring areas. It has been described that in most of the areas local community rely on these plants to cure many diseases. The use of these plants is based upon folk knowledge. It was noted that the area has important useful wild flora belonging to various families. Similar results have been found in previous studies as Saurabh et al. (2011) described that $A$. aspera is useful to cure asthma, piles, dropsy, snakebite, rheumatism and skin diseases. Amaranthus viridis $\mathrm{L}$. (Amaranthaceae) was useful for the treatment of malaria, useful for prolapse of uterus, cure urinary tract infections, useful to cure snakebite (Butt et al., 2015). During this study, it was noted that Avena sativa is considered for having antioxidant and wound healing properties, which is in accordance in the earlier findings found by Akkol et al. (2011). Calotropis procera has anti-diarrhoeal and antiinflammatory activity (Kumar and Basu, 1994). Mishra et al. (2011) described that paste of Fumaria parviflora var. indica (Hausskn.) Parsa (Papaveraceae) was useful for curing joint swellings. These fining are in accordance with the information noted during this ethnobotanical survey of Tehsil Gojra.
All the plants documented in this area showed varied values for different pharmacological attributes which have been also observed by many workers in the past. Rehman et al. (2020) conducted study to observe most common medicinal plants by local people were $C$. intybus had $100 \%$ Fidelity Level (FL) value as liver tonic and blood purifier. Highest 0.76 Informant Consensus Factor (ICF) values were noted against stomach, gastric, intestinal and digestive problems. $A$. aspera showed the highest Fidelity Level (FL) values that was $93 \%$ used gastrointestinal disorders and menstrual pain. Padmavathi et al. (2005) claimed that the high FL value showed the use of a particular species by the local people to treat a specific disease. Rehman et al. (2020) described 33 families with important weeds of the area that are used to cure various diseases including skin infection, respiratory and asthma problems, kidney, liver and heart diseases. Shinwari and Khan (2000) recorded 50 species of herbs belonging to 27 families used medicinally by people native to Margalla Hills of National Park Islamabad. From the total 50 species, only 10 species were being sold in local market. Ahmad et al. (2009) studied six important plant species having medicinal importance. A. aspera was one of them, used as purgative, laxative, diuretic, antiviral and styptic agent. Similar medicinal uses were noted among the local people of Tehsil Gojra.

Similarly, Hussain et al. (2008) reported 40 plant species from 39 genera and 32 families having medicinal properties in the Haripur region of Pakistan. They described that $A$. arabica was used as astringent, tonic, antiseptic and purgative. Habiba et al. (2016) collected 10 medicinally important plants, which have different ethnobotanical uses. Aerva javanica (Burm. f.) Juss. (Amaranthaceae) was useful to cure inflammations, abdominal worms and skin infections. Khan et al. (2014) reported that C. procera was useful to cure cough, rheumatism and skin infection. Qureshi et al. (2010) reported 63 herbs belonging to 50 genera and 29 families having medicinal importance. Matin et al. (2001) described 18 species of trees, 12 species of shrubs and 77 species of herbs being used for medicinal purpose to cure many diseases. All these studies are in accordance with the present ethnobotanical studies in Tehsil Gojra for 47 wild plant species

It has been confirmed that all the wild plants have much important role in folk medicines. Most of the local people rely on these plants based upon traditional knowledge. It has been approved in the past as well as during these surveys. These plants are also a source of raw materials for pharmaceutical industries. 


\section{Conclusions}

It was concluded that Tehsil Gojra, Punjab, Pakistan has many wild plants that is good source of folk medicine. Most of the people rely on these plants to cure diseases based upon traditional knowledge. Thus, there is a need to raise awareness among people to use these plants based upon authentic documented information.

\section{Declarations}

List of abbreviations: Not applicable.

Ethical approval and consent to participate: This study was undertaken with approval of Local participants and community of Tehsil Gojra, district Toba Tek Singh, Punjab, Pakistan.

Consent for publication: Not applicable

Conflict of interests: The authors have declared that they have no conflict of interests.

Funding: This study has not received any grant/ funding form any agencies or forum.

Authors' contributions: MT, KH and KN planned the study and surveys, $\mathrm{KH}$ and $\mathrm{GY}$ identified the plants with reference herbarium. SSA helped in write up of this paper.

\section{Literature cited}

Abbasi, Mehmood A, Shah MH, Khan MA. 2016. Wild Edible Vegetables of Lesser Himalayas. Springer, Cham, Switzerland. pp. 360

Ahmad M, Qureshi R, Arshad M. 2009. Traditional herbal remedies used for the treatment of diabetes from district Attock (Pakistan). Pakistan Journal of Botany 41(6): 2777-2782.

Akkol EK, Süntar I, Orhan IE. 2011. Assessment of dermal wound healing and in vitro antioxidant properties of Avena sativa L. Journal of Cereal Science 53: 285-90.

Alamgeer, Sharif A, Asif H, Younis W, Riaz H, Ishfaq, Bukhari A, Assiri AM. 2018. Indigenous medicinal plants of Pakistan used to treat skin diseases: a review. Chinese Medicine 13: 52-60.

Ali, SS, Hussain K, Nawaz K, Bhatti KH, Bashir Z, Nazeer A, Arif U, Jafar S, Siddiqi EH. 2020. Ethnobotanical knowledge and folk medicinal significance of the flora of district Gujrat, Punjab, Pakistan. Herba Polonica 66(1):1-14.

Butt MA, Ahmad M, Fatima A. 2015. Ethnomedicinal uses of plants for the treatment of snake and scorpion bite in Northern Pakistan. Journal of Ethnopharmacology 168: 64-181.

Cunningham AB. 2014. Applied ethnobotany:people, wild plant use and conservation. Routledge 1:165170.
Gilani AH, Rahman A. 2005. Trends in ethnopharmacology. Journal of Ethnopharmacology 100:43-49.

Hussain A, Khan MN, Iqbal Z. 2008. An account of the botanical anthelmintics used in traditional veterinary practices in Sahiwal district of Punjab, Pakistan. Journal of Ethnopharmacology 119(1):185190.

Hussain K, Shahazad A, Zia-ul-Hussnain S. 2008. An ethnobotanical survey of important wild medicinal plants of Hattar district Haripur, Pakistan. Ethnobotanical Leaflets 12:29-35.

Hussain K, Nisar MF, Majeed A. 2010. Ethnomedicinal survey for important plants of Jalalpur Jattan, district Gujrat, Punjab, Pakistan. Ethnobotanical Leaflets 7:11.

Khan N, Abbasi AM, Dastagir G. 2014. Ethnobotanical and antimicrobial study of some selected medicinal plants used in Khyber Pakhtunkhwa (KPK) as a potential source to cure infectious diseases. BMC Complementary and Alternative Medicine 14(1):122-132.

Khan AW, Khan A, Shah SMM, Ullah A, Faheem M, Saleem M. 2019. An updated list of neuromedicinal plants of Pakistan, their uses, and phytochemistry. Evidence-Based Complementary and Alternative Medicine 2019:1-28.

Kumar VL, Basu NO. 1994. Anti-inflammatory activity of the latex of Calotropis procera. Journal of Ethnopharmacology 44(2):123-125.

Levy PS, Lemeshow S. 2008. Sampling of populations. Methods and Applications. New York:John Wiley \& Sons.

Mahmood A, Mahmood A, Malik RN, Shinwari ZK. 2013. Indigenous knowledge of medicinal plants from Gujranwala district, Pakistan. Journal of Ethnopharmacology 148:714-723.

Matin A, Khan MA, Ashraf M. 2001. Traditional use of herbs, shrubs and trees of Shogran valley, Mansehra, Pakistan. Pakistan Journal of Biological Science 4:1101-7.

Minallah MN, Rafique M, Anwar MM, Mohsin M. 2016. Assessing the urban growth and morphological patterns of Gojra city, Pakistan. Sindh University Research Journal 48(2):393-398.

Mishra G, Singh P, Verma R, Kumar S. 2011. Traditional uses, phytochemistry and pharmacological properties of Moringa oleifera plant:An overview. Der Pharmacia Letter 3(2):141164.

Muhammad S, Khan Z, Cheema TA. 2009. Distribution of weeds in wheat, maize and potato fields of Tehsil Gojra, district Toba Tek Singh, Pakistan. Pakistan Journal of Weed Science Research 15(1):91-105. 
Njoroge G, Bussmann R, Gemmill B, Newton L, Ngumi V. 2004. Utilization of weed species as source of traditional medicines in central Kenya. Lyonia 7:7187.

Padmavathi B, Rath PC, Rao AR. 2005. Roots of Withania somnifera inhibit for stomach and skin carcinogenesis in mice. Evidence-Based Complementary and Alternative Medicine 2(1):99105.

Petrovic J, Stanojkovic A, Comic L, Curcic S. 2005. Antibacterial activity of Cichorium intybus. Fitoterapia 75:737-9. doi:10.1016/j.fitote.2004.05.001.

Qureshi R, Bhatti GR, Memon RA. 2010. Ethnomedicinal uses of herbs from northern part of Nara desert, Pakistan. Pakistan Journal of Botany 42(2):839-851.

Rehman A, Hussain K, Nawaz K, Arshad N, lqbal I, Ali SS, Nazeer A, Bashir Z, Jafar S, Arif U. 2020. Indigenous knowledge and medicinal significance of seasonal weeds of district Gujrat, Punjab, Pakistan. Ethnobotany Research and Applications 20(9):1-19.

Saurabh S, Camerota A, Zavotsky J. 2011. Gallstone ileus:diagnostic and therapeutic dilemma. International Journal of Case Report and Images 2:15.

Shinwari ZK. 1996. Ethnobotany in Pakistan: Sustainable and participatory approach. In Proc. 1st Training Workshop on Ethnobotany and its Application to Conservation NARC. Journal of Biological Environmental Sciences 10(2):14-25.

Shinwari MI, Khan MA. 2000. Folk use of medicinal herbs of Margalla Hills National Park, Islamabad. Journal of Ethnopharmacology 69(1):45-56.

Shinwari ZK, Gilani SS. 2003. Sustainable harvest of medicinal plants at Bulashbar Nullah, Astore (northern Pakistan). Journal of Ethnopharmacology 84(2-3):289-298.

Umair M, Altaf M, Abbasi AM. 2017. An ethnobotanical survey of indigenous medicinal plants in Hafizabad district, Punjab-Pakistan. PLoS One 12(6):e0177912. 